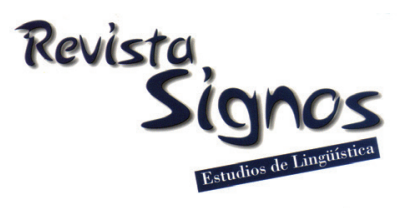

\title{
Análisis de la competencia lingüístico-discursiva escrita de los alumnos de nuevo ingreso del Grado de Maestro en Educación Primaria ${ }^{1}$
}

\section{Analysis of written linguistic and discursive competence of first year students in Primary Education Teacher Degree}

Ana María Rico Martín

Universidad de Granada

ESPAÑA

amrico@ugr.es
Dimitrinka Níkleva

Universidad de Granada

ESPAÑA

nikleva@ugr.es

Recibido: 11-II-2014 / Aceptado: 30-III-2015

\section{Resumen}

Cuando un alumno llega a la universidad, se le supone adquirido un bagaje lingüístico y lectoescritor que le permitirá la elaboración de trabajos escritos empleando un discurso académico adecuado a su nivel. No obstante, numerosas investigaciones reflejan las carencias lingüístico-discursivas del alumnado universitario. En este artículo, tras exponer estos estudios precedentes, los contenidos y competencias lingüísticas del currículo del futuro maestro y las características del discurso académico universitario, se recoge un análisis de muestras de escritura en la prueba de una materia de Lengua española de tres promociones de alumnos de nuevo ingreso en el Grado de Maestro en Educación Primaria de la Universidad de Granada (España) con el objetivo de detectary clasificar estas carencias. Los resultados de esta investigación de metodología empíricoanalítica descriptiva reflejan el mejor rendimiento de las mujeres en la asignatura que se evalúa, así como del alumnado de origen europeo, pero, principalmente, todos muestran debilidades en su expresión escrita -errores fonológicos, morfológicos, sintácticos, léxico-semánticos, ortográficos y del discurso-, errores propios de etapas educativas anteriores, sobre todo los de tipo gramatical, fruto del bajo dominio de las convenciones textuales y lingüísticas. Se concluye en la necesidad de ofrecer soluciones interdisciplinares desde la propia institución y se plantean algunas propuestas generales de prácticas discursivas escritas integradas en la formación de estos futuros docentes.

Palabras Clave: Competencia lingüística, escritura académica, carencias lingüísticodiscursivas, alumnado universitario, futuro docente. 


\begin{abstract}
When students start university, they are supposed to have already acquired a linguistic and literacy background that will allow them to write texts using an academic discourse appropriate to their level. However, most research focuses on the lack of linguistic-discourse competence in university students. After exposing these previous studies, the contents and language skills included in the curriculum of future teachers and the characteristics of university academic discourse, this paper covers an analysis of some writing samples in the tests of Spanish of three groups of first year students in the Degree in Primary Education Teacher Program from the University of Granada (Spain) in order to detect and classify these deficiencies. Results of this empiricalanalytical descriptive study show that women, as well as students from Europe, perform better in the subject being evaluated, but most of them have problems in their writing (i.e., phonological, morphological, syntactic, lexical-semantic, spelling and discourse errors), which are specific to previous educational stages, especially those of grammatical type, caused by their limited knowledge about textual and linguistic conventions. Conclusions focus on the need of interdisciplinary solutions from the institution itself. Some general proposals of written discursive practices integrated into the training of these future teachers are raised.
\end{abstract}

Key Words: Llinguistic competence, academic writing, linguistic-discursive weaknesses, university students, future teacher.

\title{
INTRODUCCIÓN
}

La adquisición de la escritura requiere del alumnado un análisis lingüístico y un trabajo riguroso, ello le da pie a plantear muchos comentarios metalingüísticos que reflejan la complejidad y problemática que la escritura genera en los jóvenes a lo largo de toda su formación, pues, como se leerá en estas páginas, los estudiantes universitarios aún cometen errores en la dimensión lingüística y textual del discurso escrito que deberían haber superado en etapas educativas previas.

Conscientes de que "la evaluación en la universidad debe ser algo más que evaluar conocimientos científicos” (Porto, 2008: 137), el docente universitario debe considerar en ella otros aspectos como competencias o habilidades que conformarán, en un futuro, un perfil profesional determinado; según tal premisa, en estas páginas se evalúa la 'competencia lingüística y discursiva académica' de estudiantes de nuevo ingreso del Grado de Maestro en Educación Primaria de la Universidad de Granada (UGR) (España) mediante el análisis de muestras textuales extraídas de una prueba presencial escrita de una asignatura de Lengua española de este Grado.

En el nivel académico universitario se dan por supuestos todos los procedimientos, técnicas y estrategias vinculados a la escritura y trabajados a lo largo de la vida escolar, 
no obstante, en la formación del futuro maestro se contemplan competencias referidas a contenidos lingüísticos comunicativos, bien sobre el propio sistema, bien sobre el uso de la lengua.

En lo que respecta a estas competencias y al tema que ocupa estas páginas, sobra decir que el futuro docente debe estar lo suficientemente capacitado para abordar la enseñanza de la escritura de textos habituales de la vida cotidiana y ejercer como escritor modelo para sus alumnos; es por ello que, por ejemplo, hay investigaciones y experiencias realizadas con escolares como escritores junto a estudiantes de Magisterio en el papel de observadores y guías de ellos en tareas de escritura en grupo (Milian, 2001).

De las asignaturas del Grado que constituyen el módulo de Enseñanza y aprendizaje de las lenguas, la investigación que aquí se presenta se enmarca en la docencia de la denominada Didáctica de la Lengua española I, del curso primero, que abarca todos los niveles atendiendo a la lengua como sistema (fonético-fonológico, morfosintáctico, léxico-semántico) y al uso de esta lengua (pragmática, sociolingüística y análisis textual).

El objetivo de esta investigación es, pues, la detección y clasificación de las carencias lingüístico-discursivas que presenta el alumnado del primer curso del Grado de Maestro en Educación Primaria de la Facultad de Educación y Humanidades de Melilla (UGR) al final del año académico correspondiente, carencias que comprometen gravemente la propia 'competencia lingüística comunicativa'.

\section{Marco teórico}

\subsection{El discurso académico en la universidad}

Cuando se dice de una persona que tiene una buena 'competencia en comunicación lingüística', se le supone desarrollada una capacidad para comprender y producir textos orales y escritos en diferentes situaciones comunicativas, con diversas intenciones y para distintos destinatarios; se alude, pues, a la habilidad para emplear diferentes géneros discursivos con sus propias normas (Adam, 1999) y en los contextos sociales adecuados o 'esferas de la actividad humana' (Bajtín, 1979). En este trabajo se acota este campo tan amplio de los géneros textuales para centrarse en el discurso académico.

Cassany, Luna y Sanz (2000) caracterizan al texto académico como un subgénero ante todo referencial y representativo, cuya finalidad es construir y transmitir el conocimiento mediante un discurso muy elaborado, con un registro formal y un lenguaje objetivo, de léxico preciso y muy específico, además de biunívoco y universal.

La capacidad lingüística dominante es, pues, exponer y presentar, de manera textual, diferentes formas de saberes a través de distintas estrategias orales y escritas, por lo que conviene que los docentes universitarios incluyan en su programación 
actividades donde se trabaje con el lenguaje científico propio de la materia, adaptado a sus alumnos y al perfil profesional para el que se forman, en la línea de lo que se hace en otros niveles educativos: la escritura colaborativa en el ámbito de las Ciencias en la ESO (Oliveras \& Sanmartí, 2011) o el análisis de la coherencia del discurso en aulas de Bachillerato (Tamayo \& Sanmartí, 2005).

De hecho, en opinión de Carlino (2003), la dificultad de lectura, y en consecuencia de escritura, que pueden tener los estudiantes universitarios se debe a que se enfrentan con nuevas culturas escritas procedentes de diferentes ámbitos de conocimiento y dirigidas a expertos en esos ámbitos y no precisamente para estudiantes. En efecto, según Rodríguez y Ruiz Bikandi (2013), el discurso científico siempre ha sido considerado como derivado natural de la actividad científica y, en consecuencia, no se ha concebido la necesidad de trabajarlo didácticamente en el aula universitaria; por esta razón, es importante que se trabajen la escritura y lectura de textos en las diferentes materias curriculares. Considérese, además, que como culmen de sus estudios el estudiante de cualquier grado tiene que demostrar, entre otras, una competencia escritora importante en el momento de realizar el Trabajo Fin de Grado (TFG), aunque Castelló, Pardo y Fuentealba (2011) señalan que pertenecería a un género textual intermedio entre el propio texto académico universitario y el texto científico dirigido a una comunidad científica.

Bien que el empleo de la escritura académica se da en muy diversos ámbitos, indudablemente es característica del universitario, por tanto, objeto de investigación y lugar común entre los docentes universitarios, ya que con resultados como los que refleja el estudio de estas páginas, requieren intervención y políticas educativas específicas (Carlino, 2007; Martí, Amat \& Jiménez, 2013).

\subsection{Antecedentes del estudio}

Las dificultades en la expresión escrita que pueden tener los alumnos de estudios superiores no han pasado desapercibidas a los propios docentes, como lo demuestran numerosas investigaciones de distintas titulaciones, universidades y países, en este caso, de habla española, que se han realizado en las últimas décadas.

Interesan ahora especialmente las que evalúan la calidad de la propia escritura académica. Algunas de estas señalan las características generales del discurso académico, como ocurre con las de Castelló et al. (2011), Catuogno, Mallo y Rivarola (2003), Dolz, Gagnon, Mosquera y Sánchez Abdchi (2013), Marín y Morales (2004), Martí et al. (2013), Morales y Espinoza (2005), o Nigro (2006). Tapia, Burdiles y Arancibia (2003) también estudian los aspectos más relevantes del informe universitario pero evalúan su estructura y su calidad, siendo esta última dimensión la que coincide con los niveles lingüísticos que se examinan en estas páginas. 
En otros casos, el análisis del discurso recae en niveles concretos, por ejemplo, en la ortografía, como en los trabajos de Argáez, Fernández, Martínez, Sandoval y Ortiz (2010), Medina (1994), Morales y Hernández (2004) o Sánchez Lozano (2007).

Además, estos cuatro trabajos señalan que las muestras de escritura universitaria estudiadas presentan errores propios de etapas educativas anteriores, de la misma forma que Martí et al. (2013) mencionan la incorrecta separación de sílabas de una palabra o Dolz et al. (2013) insisten en la confusión de categorías gramaticales de algunos escritos.

Pero la mayor frecuencia de errores se encontró sobre todo entre las cualidades textuales referidas a la coherencia y la cohesión (Catuogno et al., 2003; Morales \& Hernández, 2004; Morales \& Espinoza, 2005; Tamayo \& Sanmartí, 2005; Sánchez Avendaño, 2006) y, en menor grado, en la adecuación del discurso, cuando los alumnos mostraron rasgos informales en un texto académico (Catuogno et al., 2003; Morales \& Espinoza, 2005; Sánchez Avendaño, 2005, 2006; Nigro, 2006).

Se observan en los autores mencionados que los errores aludidos son comunes en gran parte de las investigaciones, aunque algunos insistan más en algún aspecto concreto. Los participantes de nuestro estudio también presentan estos tipos de errores como se verá en las líneas siguientes.

\section{Marco metodológico}

Respecto al diseño de investigación, se ha empleado una metodología empíricoanalítica con un diseño ex-post-facto de tipo descriptivo (Rodríguez \& Valldeoriola, 2009), de los más utilizados en el ámbito educativo pues favorece tanto la descripción de la realidad como la organización de las variables objeto de estudio y el análisis de sus relaciones. En este caso, al aunar datos cuantitativos y cualitativos, facilita la clasificación y cuantificación de la tipología de carencias lingüístico-discursiva del alumnado participante.

\subsection{Contexto y participantes}

Si se parte del contexto más amplio de la investigación, es necesario señalar que la Facultad de Educación y Humanidades donde estudian los participantes pertenece al Campus de la UGR sito en la Ciudad Autónoma de Melilla, de 84509 hab. $^{2}$, enclavada al norte del continente africano y rodeada por fronteras con Marruecos y el Mar Mediterráneo; en sus $12,3 \mathrm{Km}^{2}$ cohabitan cuatro etnias que determinan la idiosincrasia de la población (de origen peninsular o europeo ${ }^{3}$, bereber, hebreo e hindú). Los dos grupos de población mayoritaria son el de origen peninsular y el de los bereberes.

La Facultad es un centro universitario pequeño que cuenta con un número aproximado de 360 alumnos de Grado. Casi todo su alumnado es de nacionalidad 
española y emplea como lengua frecuente y vehicular de la enseñanza la oficial de la ciudad: el español, aunque parte de los alumnos de origen bereber mantiene su lengua materna oral tamazigh o mazigio, procedente de una lengua milenaria de origen camitosemítica, con su variante de habla taqer'art.

El número de alumnos del Grado de Maestro en Educación Primaria era de 178 durante el curso 2012-2013. La muestra de este estudio está constituida por los 123 alumnos que cursaron la materia de primer curso Didáctica de la Lengua Española I desde la implantación del Grado en el año 2010-2011, por lo que son tres promociones del alumnado cuya competencia lingüística se analiza en estas páginas. La descripción detallada de esta muestra se recoge en la Tabla 1.

Tabla 1. Descripción de la muestra.

\begin{tabular}{|c|c|c|c|}
\hline Año académico & Sexo & Edad & Origen cultural \\
\hline $\begin{array}{l}2010-2011 \\
N=44\end{array}$ & $\begin{array}{l}\text { Hombre: } 40,9 \% \\
\text { Mujer: } 59,1 \%\end{array}$ & $\begin{array}{l}\text { 19-20: } 95,5 \% \\
\text { 21-25: } 4,5 \% \\
>25: 0 \%\end{array}$ & $\begin{array}{l}\text { Peninsular: } 79,5 \% \\
\text { Bereber: } 20,5 \% \\
\text { Hebreo: } 0 \%\end{array}$ \\
\hline $\begin{array}{l}2011-2012 \\
N=40\end{array}$ & $\begin{array}{l}\text { Hombre: } 45 \% \\
\text { Mujer: } 55 \%\end{array}$ & $\begin{array}{l}\text { 19-20: } 87,5 \% \\
21-25: 7,5 \% \\
>25: 5 \%\end{array}$ & $\begin{array}{l}\text { Peninsular: } 65 \% \\
\text { Bereber: } 35 \% \\
\text { Hebreo: } 0 \%\end{array}$ \\
\hline $\begin{array}{l}2012-2013 \\
N=39\end{array}$ & $\begin{array}{l}\text { Hombre: } 35,9 \% \\
\text { Mujer: 64,1\% }\end{array}$ & $\begin{array}{l}\text { 19-20: } 76,9 \% \\
21-25: 5,1 \% \\
>25: 17,9 \%\end{array}$ & $\begin{array}{l}\text { Peninsular: } 74,4 \% \\
\text { Bereber: } 23,1 \% \\
\text { Hebreo: } 2,6 \%\end{array}$ \\
\hline $\begin{array}{l}\text { Totales } \\
\mathrm{N}=123\end{array}$ & $\begin{array}{l}\text { Hombre: } 40,7 \% \\
\text { Mujer: } 59,3 \%\end{array}$ & $\begin{array}{l}\text { 19-20: } 87 \% \\
21-25: 5,7 \% \\
>25: 7,3 \%\end{array}$ & $\begin{array}{l}\text { Peninsular: } 73,2 \% \\
\text { Bereber: } 26 \% \\
\text { Hebreo: } 8 \%\end{array}$ \\
\hline
\end{tabular}

Se observa el predominio de las alumnas y de la edad media de acceso a la universidad (19 o 20 años), considerando que es una asignatura de primer curso.

En lo que concierne al origen cultural, obsérvese que destaca el alumnado de procedencia peninsular, aunque el bereber es cada vez más numeroso, no obstante, en el curso 2012-2013 sufrió un retroceso respecto al anterior.

\subsection{Variables}

Además de las variables independientes que sirvieron para caracterizar a los participantes (sexo, edad y origen cultural) y el rendimiento académico en la asignatura en cuestión, la investigación maneja como variable principal la 'competencia lingüística’ del alumnado, analizada según los diferentes niveles lingüísticos: fonológico, morfológico, sintáctico, léxico-semántico y discursivo, como se observa en la Figura 1. 


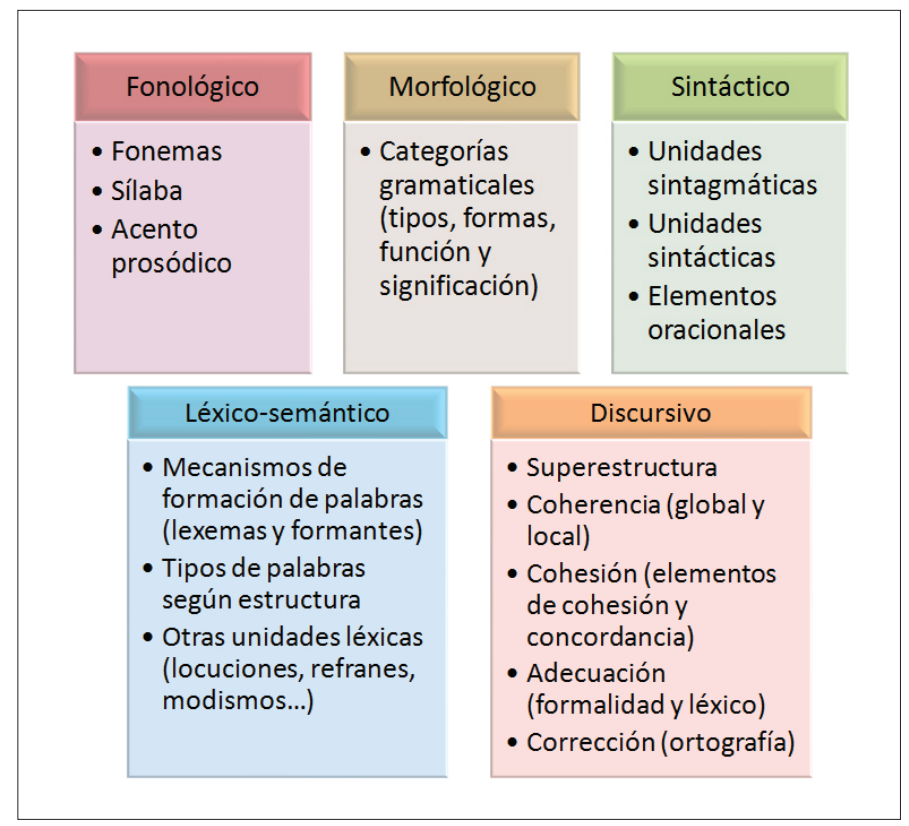

Figura 1. Niveles lingüísticos como categorías de análisis.

La tarea del alumnado en cada uno de estos niveles consiste en identificar, definir y caracterizar sus unidades específicas según lo trabajado en la asignatura.

Estos niveles de análisis estaban ya predeterminados en tanto que son los que corresponden a los contenidos de la asignatura y se mostraban diferenciados en la prueba excepto lo que corresponde al nivel discursivo, que se extraía de las preguntas de desarrollo.

Se han tomado como indicadores del nivel discursivo una adaptación de los empleados por Marín y Morales (2004) y por Tapia et al. (2003) en la pauta de evaluación para informes académicos (Dimensión 7. Calidad del escrito).

De esta forma, se ofrece una perspectiva integral de la composición escrita: conocimientos de normas y reglas de producción textual (Morales \& Espinoza, 2005). El grado de dominio de los niveles mencionados viene determinado por la cantidad y la calidad de los errores que los alumnos presentan en cada uno.

\subsection{Técnicas e instrumento de recogida de datos}

Como instrumento de recogida de la información se empleó una prueba presencial escrita con secciones referidas a cada nivel lingüístico mencionado:

a) Nivel fonológico. Transcripción fonológica de un texto determinado, análisis de las sílabas y de los fonemas de palabras extraídas del texto. 
b) Nivel morfológico. Análisis morfológico de un texto, en el que se debían caracterizar todas las categorías gramaticales que aparecían.

c) Nivel sintáctico. Análisis sintagmático y sintáctico de un texto.

d) Nivel léxico-semántico. Caracterización de una serie de unidades léxicas (locuciones, refranes, frases proverbiales, modismos), además de actividades de descomposición y clasificación de palabras según sus formantes.

e) Nivel discursivo. Análisis de la expresión escrita del alumnado manifiesta en las preguntas de desarrollo temático. En este nivel se estudian los rasgos propios del alumno como escritor, por tanto, va más allá de las cuestiones lingüísticas y estrictamente gramaticales trabajadas en el transcurso de la materia.

Aunque lo adecuado es trabajar los niveles de forma integrada en los textos, para delimitar mejor los aciertos y errores de cada uno de ellos se mantuvieron separados.

\subsection{Procedimiento de recogida de la información}

El análisis de la competencia lingüística mostrada por los alumnos se realizó tras cuatro meses de trabajo en la asignatura de Didáctica de la Lengua Española I, en tres sesiones semanales de 60 minutos. Al término de los cuatro meses, los alumnos realizaron la prueba individual mencionada en un tiempo máximo de dos horas.

\section{Análisis de datos y resultados de la investigación}

Para el análisis de los textos del alumnado se realizó una revisión bibliográfica con el fin de encontrar un método idóneo para clasificar los errores lingüísticos, se comprobó que no existe consenso entre los estudiosos referidos entre los antecedentes que permitiera decidirse por una tipología concreta, por lo que se acordó como procedimiento de análisis la agrupación de los distintos errores encontrados en los textos según las dimensiones mencionadas. En lo referente al nivel discursivo, se han considerado la dimensión 7 de la pauta de Tapia et al. (2003) y los indicadores de Marín y Morales (2004).

En un primer momento del análisis -realizado mediante el programa informático Statistical Package for Social Sciences (SPSS) - se utilizó una estadística descriptiva para caracterizar la muestra participante, las frecuencias y porcentajes respecto a algunas variables. Después, se ha hecho uso de una estadística inferencial que ha permitido comparaciones bivariables. El número de alumnos participantes favoreció el empleo de estadísticos paramétricos. El valor del 'nivel de significación' observado es menor que, 05 . 


\subsection{El rendimiento académico en la asignatura Didáctica de la Lengua Española I}

Este rendimiento venía reflejado mediante la calificación obtenida por el alumnado al acabar la asignatura. Los valores numéricos de cada calificación son los siguientes: Suspenso: 0-4,9; Aprobado: 5-6,9; Notable: 7-8,9; Sobresaliente: 9-10.

La media del alumnado del curso es ${ }^{\bar{x}}=5,83$. En el Gráfico 1 se observa el predominio de los alumnos con Aprobado, si bien es muy importante el alto porcentaje de alumnos suspensos.

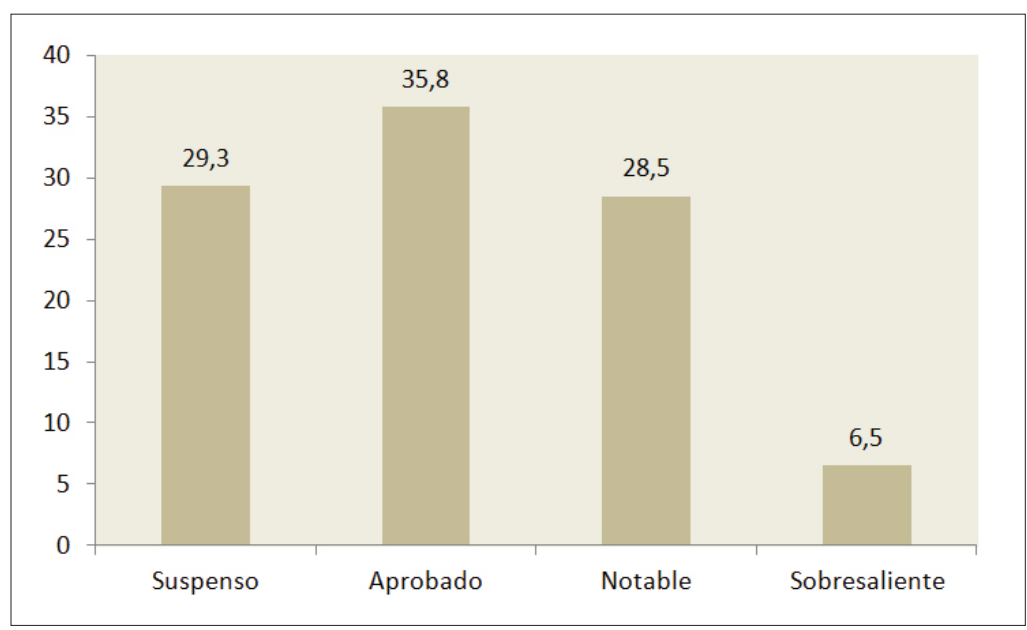

Gráfico 1. Porcentaje de calificaciones en Didáctica de la Lengua Española I.

Con la intención de conocer el rendimiento dependiendo del sexo del alumnado, se empleó el estadístico de contraste ' $t$ de Student', que señaló diferencias significativas entre las calificaciones $(t=-3,45$; Sig. $=, 001)$, en este caso las mujeres obtienen mejor puntuación: ${ }_{\text {Alumnas }}^{\bar{x}}=6,31$ (para $\mathrm{N}_{\text {Alumnas }}=73$ ) y ${ }_{\text {Alumnos }}=5,13\left(\right.$ para $\left.\mathrm{N}_{\text {Alumnos }}=50\right)$.

Al comparar mediante un 'ANOVA' las calificaciones atendiendo a la edad de los alumnos, se observó que no existían diferencias significativas entre los tres sectores de distribución $(F=, 482$; Sig. $=, 619):{ }^{\bar{x}}{ }_{19-20}=5,845 ;{ }_{21-25}=5,214 ;{ }^{\bar{x}}{ }_{25}=6,167$.

En el caso de la procedencia cultural del alumnado, el cotejo de medias refleja que el resultado es estadísticamente significativo $(F=3,06$; Sig. = ,051), los alumnos de origen peninsular obtienen calificaciones algo mejores que los de ascendencia bereber: ${ }_{\text {Peninsular }}=6,071 ;{ }_{\text {Bereber }}=5,125$.

\subsection{Nivel fonológico}

Al interpretar los resultados del alumnado en el nivel fonológico de la prueba (véase Tabla 2), llama la atención que aún haya estudiantes que no dominen la 
separación y clasificación de las sílabas españolas (4,9\%); se trata de un contenido trabajado en el currículo escolar desde el segundo ciclo de la Educación Primaria. Respecto a la identificación de los fonemas, procedimiento nuevo para el alumnado, crece la frecuencia de jóvenes con dificultades en ello.

Tabla 2. Frecuencia de los principales errores relativos al nivel fonológico.

\begin{tabular}{|l|c|c|}
\hline \multicolumn{1}{|c|}{$\begin{array}{c}\text { Dificultades } \\
\text { Nivel fonológico }\end{array}$} & Frecuencia & Porcentaje \\
\hline Ninguna & 93 & 75,6 \\
\hline Fonemas & 24 & 19,5 \\
\hline Sílabas & 6 & 4,9 \\
\hline Total & 123 & 100,0 \\
\hline
\end{tabular}

\subsection{Nivel morfológico}

Dada la diversidad de categorías gramaticales con las que debían trabajar los alumnos, para realizar el análisis se distribuyeron en cuatro bloques: 1) componentes del sintagma nominal, 2) componentes del verbal, 3) elementos relacionantes y 4) un bloque extraído del sintagma nominal y dedicado a los pronombres que mayores dificultades suponen al alumnado: los personales y los indefinidos. La Tabla 3 aglutina los resultados.

Tabla 3. Frecuencia de los principales errores relativos al nivel morfológico.

\begin{tabular}{|l|l|l|l|}
\hline \multicolumn{2}{|c|}{$\begin{array}{c}\text { Dificultades } \\
\text { Nivel morfológico }\end{array}$} & Frecuencia & Porcentaje \\
\hline \multirow{5}{*}{ Bloque 1 } & Ninguna & 68 & 55,3 \\
\cline { 2 - 4 } & Determinantes & 49 & 39,8 \\
\cline { 2 - 4 } & Adjetivos calificativos & 6 & 4,9 \\
\cline { 2 - 4 } & Total & 123 & 100,0 \\
\hline \multirow{5}{*}{ Bloque 2 } & Ninguna & 93 & 75,6 \\
\cline { 2 - 4 } & Verbo & 22 & 17,9 \\
\cline { 2 - 4 } & Adverbios & 8 & 6,5 \\
\cline { 2 - 4 } & Total & 123 & 100,0 \\
\hline \multirow{5}{*}{ Bloque 4 3} & Ninguna & 72 & 58,5 \\
\cline { 2 - 4 } & Preposición & 2 & 1,6 \\
\cline { 2 - 4 } & Conjunción & 49 & 39,8 \\
\cline { 2 - 4 } & Total & 123 & 100,0 \\
\hline & Ninguna & 63 & 14,6 \\
\cline { 2 - 4 } & Pronombres personales & 18 & 100,0 \\
\cline { 2 - 4 } & Pronombres indefinidos & 42 & \\
\cline { 2 - 4 } & Total & 123 & \\
\hline
\end{tabular}


Se observa que el bloque del grupo verbal (2..$\left.^{\circ}\right)$ es el que más dominan los alumnos. Las categorías lingüísticas que mejor identifican son la preposición, el adjetivo calificativo y el adverbio (aparte del sustantivo, donde no hubo errores). Hay que insistir en que las categorías que se mencionan están presentes también entre los contenidos de la Educación Primaria, por lo que deberían dominarse en la etapa universitaria.

Los elementos que más dificultades conllevan son, con el mismo porcentaje de errores (39,8\%), los determinantes y la conjunción, en particular, los indefinidos y los pronombres relativos, en concreto 'que', elemento subordinante que confunden frecuentemente con la conjunción de valor completivo.

\subsection{Nivel sintáctico}

Para analizar este nivel, también se recurrió a la distribución en bloques de aquellos elementos en los que mayores errores cometía el alumnado: 1) componentes del sujeto, 2) componentes del predicado y 3) tipos de oraciones subordinadas.

Tabla 4. Frecuencia de los principales errores relativos al nivel sintáctico.

\begin{tabular}{|l|l|c|c|}
\hline \multicolumn{2}{|l|}{$\begin{array}{l}\text { Dificultades } \\
\text { Nivel sintáctico }\end{array}$} & Frecuencia & Porcentaje \\
\hline \multirow{5}{*}{ Bloque 1 } & Ninguna & 116 & 94,3 \\
\cline { 2 - 4 } & Sujeto/predicado & 5 & 4,1 \\
\cline { 2 - 4 } & Determinantes & 2 & 1,6 \\
\cline { 2 - 4 } & Total & 123 & 100,0 \\
\hline \multirow{5}{*}{ Bloque 2 } & Ninguna & 77 & 62,6 \\
\cline { 2 - 4 } & CD / Supl. / CI & 36 & 29,3 \\
\cline { 2 - 4 } & $\begin{array}{l}\text { CPrvo. / Atr. / CC } \\
\text { Bloque 3 } 3\end{array}$ & 8 & 6,5 \\
\cline { 2 - 4 } & Modo & 2 & 1,6 \\
\cline { 2 - 4 } & CC & 123 & 100,0 \\
\hline & Total & 72 & 58,5 \\
\cline { 2 - 4 } & Singuna & 12 & 9,8 \\
\cline { 2 - 4 } & Subord. sustantiva & 34 & 4,1 \\
\cline { 2 - 4 } & Subord. adjetiva & 5 & 100,0 \\
\cline { 2 - 4 } & Total & 123 & \\
\hline
\end{tabular}

Nota: $\mathrm{CD}=$ complemento directo; Supl. $=$ suplemento; $\mathrm{CI}=$ complemento indirecto; CPrvo.= complemento predicativo; Atr. $=$ atributo; $\mathrm{CC}=$ complemento circunstancial.

Se aprecia en la Tabla 4 que el grupo del sujeto es el que mejor dominan, aunque algún alumno tenga problemas en los casos de oraciones pasivas reflejas donde confunden el sujeto con el CD al no respetarse el orden sintáctico canónico (sujetoverbo-predicado) ni la concordancia de sujeto-verbo, ejemplo de ello es: “...de como se aprende y controla los procesos de aprendizaje" (incluido error de acentuación 
sobre la partícula interrogativa). Igualmente, hay falta de concordancia en casos como "los conocimientos, destrezas y actitudes de esta competencia permite expresar pensamientos..."; en cambio, son las oraciones subordinadas las unidades sintácticas de mayor dificultad, sobre todo las adjetivas de relativo; recuérdese que precisamente eran los pronombres relativos los que mayores errores suponían en estos alumnos en tanto que son elementos subordinantes.

Asimismo, se destaca que el 29,3\% tiene problemas en distinguir el CD del CI cuando son pronominales y, también, confunden el primero con el suplemento.

\subsection{Nivel léxico-semántico}

La Tabla 5 muestra el estudio de las principales dificultades del componente léxico-semántico de las pruebas. En términos generales, estos elementos no plantean grandes problemas para los alumnos, no obstante, un 11,4\% es no es capaz de distinguir los formantes de las palabras, sobre todo en el caso de los verbos, cuando deben identificar los de tiempo y aspecto, o la vocal temática.

Las locuciones y su tipología también resultan difíciles para el 12,2\% del alumnado, concretamente, las adjetivas y las adverbiales.

Tabla 5. Frecuencia de los principales errores relativos al nivel léxico-semántico.

\begin{tabular}{|l|c|c|}
\hline $\begin{array}{c}\text { Dificultades } \\
\text { Nivel léxico-semántico }\end{array}$ & Frecuencia & Porcentaje \\
\hline Ninguna & 94 & 76,4 \\
\hline Formantes palabras & 14 & 11,4 \\
\hline Locuciones & 15 & 12,2 \\
\hline Total & 123 & 100,0 \\
\hline
\end{tabular}

\subsection{Nivel discursivo}

Para reflejar los principales resultados de este nivel, las características textuales y sus comentarios se han distribuido según las categorías que aparecen en la Tabla 6. 
Tabla 6. Análisis del nivel discursivo.

\begin{tabular}{|c|c|}
\hline Superestructura & Comentario \\
\hline Tipo de texto & $\begin{array}{l}\text { En general, hay cierto dominio de las características del texto expositivo académico. Predominan las ora- } \\
\text { ciones enunciativas con estructura bimembre y el orden sintáctico canónico (sujeto-verbo-predicado). }\end{array}$ \\
\hline $\begin{array}{l}\text { Organización } \\
\text { interna del texto }\end{array}$ & $\begin{array}{l}\text { Las ideas se expresan en disposición lineal, con escasa interrelación entre ellas, reflejada en el uso de la } \\
\text { yuxtaposición y de conectores aditivos. }\end{array}$ \\
\hline $\begin{array}{l}\text { Progresión } \\
\text { temática }\end{array}$ & $\begin{array}{l}\text { El 33\% del alumnado presenta ciertas dificultades en la progresión temática, inserta en el texto elementos } \\
\text { que alteran la coherencia global. Coincide con el alumnado con bajo dominio de los contenidos temáticos } \\
\text { de la materia. }\end{array}$ \\
\hline Cohe & Comentario \\
\hline $\begin{array}{l}\text { Coher } \\
\text { global }\end{array}$ & $\begin{array}{l}\text { Aunque el } 61,8 \% \text { no presenta problemas graves de coherencia, solo el } 14,63 \% \text { muestra una coherencia } \\
\text { global aceptable, con un discurso bien estructurado y cohesionado, empleando una variedad de recursos } \\
\text { conectivos del español. El resto establece interrelaciones simples entre las principales ideas, sobre todo } \\
\text { considerando que los temas de desarrollo son muy concretos, y se limitan a exponer su conocimiento sin } \\
\text { ideas adicionales. } \\
\text { El 16,3\% muestra dificultades importantes en este indicador, en muchas ocasiones emplea un lenguaje } \\
\text { tautológico. }\end{array}$ \\
\hline Coherencia local & $\begin{array}{l}\text { Predominan los textos con coherencia local, en muchos casos de tipo funcional cuando se emplean } \\
\text { proposiciones como explicaciones o, sobre todo, ejemplos. } \\
\text { Se observa coherencia interna en los párrafos (macroestructura), facilitada por la corta extensión de estos. }\end{array}$ \\
\hline Cohe & Comentario \\
\hline $\begin{array}{l}\text { Empleo de co- } \\
\text { nectores }\end{array}$ & $\begin{array}{l}\text { Hay subutilización de recursos de conexión, se repiten los mismos. } \\
\text { Predominan la yuxtaposición y los conectores aditivos ('y', 'también', 'además'), seguidos por el contraar- } \\
\text { gumentativo ('pero'), los causales ('porque', 'ya que', 'debido a') y el de finalidad ('para). } \\
\text { Otros conectores menos frecuentes son los marcadores ordenadores ('en primer lugar', 'por un lado') y el } \\
\text { reformulador recapitulativo ('por último') es de los menos usados. } \\
\text { Es de destacar que el } 100 \% \text { de los alumnos emplea el mismo operador argumentativo de ejemplificación } \\
\text { ('por ejemplo') ya que se les pide que ejemplifiquen sus respuestas. }\end{array}$ \\
\hline $\begin{array}{l}\text { Uso } \\
\text { punt }\end{array}$ & $\begin{array}{l}\text { El 17,1\% comete errores de puntuación. Destaca la omisión de comas (,) enmarcando un inciso o en pár- } \\
\text { rafos extensos, y lo contrario, añadirla entre el sujeto y el predicado. } \\
\text { Tampoco es infrecuente encontrar oraciones interrogativas o exclamativas carentes del primer signo, } \\
\text { ejemplo también de falta de adecuación textual por la influencia de la escritura abreviada de los mensajes } \\
\text { electrónicos. } \\
\text { Omisión generalizada de los dos puntos (:) y el punto y coma (;). }\end{array}$ \\
\hline Conce & $\begin{array}{l}\text { En general, hay pocos errores de concordancia, no los hay en las relaciones temporales, pues se emplea } \\
\text { ante todo el modo indicativo, el uso del condicional y el subjuntivo es mínimo. } \\
\text { Los errores encontrados son de falta de concordancia en el número del sujeto y del verbo correspondi- } \\
\text { ente, frecuente en oraciones pasivas reflejas, o de género entre el pronombre y el referente }(10 \%) \text {. }\end{array}$ \\
\hline Adecuación & Comentario \\
\hline $\begin{array}{l}\text { Formalidad del } \\
\text { escrito }\end{array}$ & $\begin{array}{l}\text { El } 21,9 \% \text { del alumnado no respeta el registro formal propio del texto académico: cae en solecismos, co- } \\
\text { loquialismos, vulgarismos y abreviaciones: "bajo mi punto de vista", "debemos de tener en cuenta que...", } \\
\text { "contra más se expresa el niño en su lenguaje...", "[pragmática] es una disciplina q se interesa por el modo } \\
\text { en q el contexto influye en el sigdo. de un mensaje." }\end{array}$ \\
\hline Pertinencia léxica & $\begin{array}{l}\text { El 4\% emplea voces inexistentes ('parasintetización' por 'parasíntesis') o fuera de contexto ('inaugurar el } \\
\text { vídeo' por 'comenzar el vídeo'). }\end{array}$ \\
\hline Corrección & Comentario \\
\hline $\begin{array}{l}\text { Ortografía de las } \\
\text { letras }\end{array}$ & $\begin{array}{l}\text { El } 83,7 \% \text { no comete errores de este tipo. } \\
\text { El } 4,9 \% \text { confunde 'b' y 'v'. } \\
\text { El } 4,1 \% \text { tiene problemas con ' } h \text { ' ('la zona geográfica donde se hubique el hablante”). } \\
\text { Un } 7,3 \% \text { tiene dificultades con grafías varias: verbos en '-ger' escritos con ' } j \text { ', 's' en lugar de 'x' y algún } \\
\text { caso de 'y' representando al fonema consonántico lateral palatal } / \lambda / \text {. }\end{array}$ \\
\hline Acent & e el acento ortográfico, el error gráfico más común del \\
\hline
\end{tabular}


Como ejemplo de dos muestras de discurso, véase el contraste de los fragmentos de dos alumnos (A1 y A2) que contestan a la misma pregunta sobre el bilingüismo:

"En muchos países se hablan varias lenguas. Hay personas que hablan varias lenguas y se les denomina bilingües. El bilingüismo es la presencia de varias lenguas distintas en un mismo lugar. Hoy en día es muy importante aprender y saber varias lenguas además de nuestra lengua materna, esta capacidad nos abrirá más puertas.” (A1)

"Podemos definir el bilingüismo, o más bien personas bilingües, como aquellas que tienen una buena competencia lingüística en dos lenguas. Generalmente, el bilingüismo se da de forma automática en regiones geográficas donde coexisten dos lenguas, es el caso de Cataluña (castellano y catalán).” (A2)

En A1 se observa la idea introductoria del fenómeno del bilingüismo, su definición y consecuencia positiva, con un predominio de la yuxtaposición formal de cuatro grupos oracionales, tres de ellos reiterativos, el empleo de dos conectores aditivos (' $y$ ', 'además de') y una oración subordinada adjetiva (introducida por el relativo 'que').

En cambio, A2 presenta un texto más elaborado y con una coherencia global algo mayor, a pesar de la brevedad del fragmento seleccionado; hay dos oraciones principales, en la primera se define el fenómeno y en la segunda se amplía el concepto con lo que se interpreta como un marcador comentador ('generalmente'); asimismo, se hace uso de un reformulador de rectificación ('más bien') y otro explicativo ('es el caso de'), además de dos subordinadas adjetivas de relativo (con 'que' y 'donde'). En este texto se reflejan mejor las relaciones lógicas entre las ideas expresas y las estructuras sintácticas, sin dejar de ser una estructura lineal.

\section{Discusión de los resultados}

Al considerar las variables independientes que caracterizaban a la muestra, se observaron diferencias significativas a favor de las mujeres respecto a la relación del rendimiento académico y el sexo del alumnado. Un resultado similar se obtuvo en el estudio de Weissa, Kemmlera, Deisenhammerd, Fleischnackera y Delazerc (2003), que evaluaba la magnitud de la diferencia entre sexos en cuanto a funciones verbales y visoespaciales; se concluyó, entonces, que las mujeres tenían mejor rendimiento en la mayoría de los tests verbales. También se halló una relación en una investigación de Mías y Ayllón (1998, citado por Robledo \& Ayllón, 2003). con alumnos universitarios de nuevo ingreso al emplear las pruebas de lenguaje incluidas en la batería DAT (Differential Aptitudes Test).

Posteriormente, Echavarri, Godoy y Olaz (2007) repitieron el estudio con tres promociones de alumnos de nuevo ingreso y concluyeron que los varones obtuvieron mejores puntuaciones en razonamiento verbal mientras que las mujeres lo obtuvieron 
en los test de ortografía y lenguaje. Igual ocurrió con el rendimiento académico de los estudiantes, las mujeres obtuvieron promedios superiores a los varones. Otros estudios similares como los de Colom y García-López (2002) o Codorniú-Raga y Vigil-Colet (2003) reflejaron tendencias parecidas.

En lo que concierne a la edad del alumnado y su relación con el rendimiento académico, no existen diferencias significativas, igual resultó en el estudio de Weissa et al. (2003), donde también hubo escasa diversidad en esta misma variable.

En cuanto a la cultura de origen, no hay datos en el entorno universitario concreto de estos alumnos que permitan comparar los resultados, no ocurre lo mismo en otros niveles educativos, donde el rendimiento académico del grupo bereber es inferior al de origen peninsular (Mesa, 2000; Mesa \& Sánchez, 1996; Sánchez, 2010), como ocurre en este estudio, aunque en el transcurso de la escolaridad obligatoria y por las características de un alumnado que llega al nivel universitario, las diferencias académicas se reducen entre ambos grupos sociales.

Hay que señalar al respecto que el alumnado bereber se va introduciendo poco a poco en el ámbito universitario, pues siempre ha habido una tendencia general a buscar una profesión o simplemente dejar de estudiar nada más concluir la educación obligatoria. De hecho, como asegura el estudio del Ministerio de Educación español sobre el abandono escolar temprano en Ceuta y en Melilla, el perfil del estudiante que abandona es de origen bereber, de entre 16 y 17 años de edad, usa la lengua española frecuentemente y su rendimiento académico es escaso, como lo es su hábito lector, lo que le genera grandes dificultades de comprensión oral y escrita (Sánchez, 2010). Esto ha supuesto que durante muchos años este alumnado no siguiera los estudios superiores, de ahí que no haya investigaciones sobre su situación fuera de las etapas educativas obligatorias.

Adentrándonos en el objeto principal de la investigación, las carencias lingüístico-discursivas del alumnado de $1 .^{\circ}$ de Grado, se han observado errores que deberían haberse subsanado en etapas educativas anteriores, sobre todo en la de Primaria (Martí et al., 2013), como son las que atañen a la segmentación de la palabra en sílabas, la diferenciación de categorías gramaticales (error en universitarios destacado también por Dolz et al., 2013), de unidades sintácticas, o de ortografía; dejando para Secundaria lo referente a la subordinación de oraciones, problema que también encontraron Morales y Espinoza (2005).

Faltas de ortografía grafofónica, aunque son las menos frecuentes en este caso universitario, también las tratan similares en no pocas investigaciones como las de Medina (1994), Morales y Hernández (2004), Morales y Espinoza (2005), Sánchez Avendaño (2006), Sánchez Lozano (2007), Sánchez Abchi, Diuk y Borzone (2009), entre otros. Si se hace referencia, además, a los problemas de acentuación y de puntuación, se añaden los estudios de Catuogno et al. (2003) y Nigro (2006). 
En el caso de la redacción, se hallaron dificultades a la hora de dotar de coherencia y cohesión a los textos, parecidas a las que señalan Catuogno et al. (2003), Morales y Hernández (2004), Morales y Espinoza (2005), Tamayo y Sanmartí (2005), Sánchez Avendaño $(2005,2006)$. A esto se une el hecho de no reconocer el propio papel de alumno universitario ni la situación en la que están produciendo el escrito, que, además, pertenece al discurso académico; ello lo lleva a no reflexionar sobre el destinatario del documento -el docente- y a cometer errores de adecuación, empleando una mezcla de lenguaje formal e informal, o de registro oral y escrito, destacado también por Catuogno et al. (2003), Morales y Espinoza (2005), Nigro (2006) y Sánchez Avendaño (2005, 2006).

Se observa, pues, que las lagunas y errores en la producción escrita de los participantes en este estudio son de características similares a los recogidos en las investigaciones referenciadas, aun procediendo de un contexto muy diferente. Ello es útil para concienciar a la comunidad universitaria de la importancia de trabajar la lengua en el transcurso de los estudios superiores y no dar por adquirida la 'competencia lingüístico-discursiva' en las etapas anteriores, como se insiste en las conclusiones de este trabajo.

\section{CONCLUSIONES}

En estas páginas se ha descrito una investigación de carácter práctico, orientada al diagnóstico de problemas de escritura del alumnado con el fin de que, a partir de sus resultados y como derivación de este trabajo, se diseñe posteriormente una intervención pedagógica sobre el discurso escrito de este alumnado universitario.

Entendidos en el tema (entre ellos, Tapia et al., 2003; Morales \& Hernández, 2004; Carlino, 2007) advierten que, cuando se detecta que los estudiantes de nuevo ingreso en la universidad muestran claras deficiencias en su competencia lingüística comunicativa, es imprescindible que las instituciones universitarias diseñen y pongan en marcha acciones en forma de cursos o talleres, donde se originen situaciones de aprendizaje en las que los alumnos practiquen la escritura académica, "con buen manejo de la ortografía y la normativa idiomática” (Sánchez Avendaño, 2006: 77).

El empleo correcto de la lengua vehicular de la enseñanza es fundamental en la formación de los futuros docentes, no solo porque será su instrumento de trabajo en el aula escolar, sino porque, de forma más inmediata, el grado de dominio del español de estos estudiantes repercutirá indiscutiblemente en la adquisición de las competencias y capacidades vinculadas al resto de las materias que constituyen su currículo formativo. En nuestro contexto más concreto, los resultados obtenidos en esta investigación en la Facultad de Educación y Humanidades de Melilla motivaron que el Decanato y el Equipo Docente del Grado se plantearan, a través del Gabinete de Orientación Universitaria (GOU), una serie de talleres englobados en el llamado Kit de supervivencia para el alumnado universitario. Dos de ellos se centran en la redacción 
de un trabajo académico y en escribir y hablar correctamente para corregir los vicios lingüísticos de los alumnos de esta edad, mediante una propuesta didáctica constructivista, basada en la escritura y la oralidad como proceso, en la que el alumno se introduce en ese proceso expresivo, ideando, produciendo, revisando y corrigiendo diversos borradores de los textos convenidos, en la línea de lo que sugieren Marín y Morales (2004) o Barboza, Aguirre y Peña (2008).

Hay que crear la conciencia en estos estudiantes de la importancia de considerar la escritura como proceso, con unos propósitos y funciones específicos para el medio académico, laboral y social (Barboza et al., 2008), y hacia un tipo determinado de lector. Además, es necesario que una buena competencia en escritura no solo académica sino de cualquier tipo pase por conocer, dominar y practicar correctamente la normativa de la lengua vehicular de la enseñanza, en este caso, la española, como aseguran Arnoux, Di Stefano y Pereira (2002) y Nigro (2006).

En el contexto concreto de los alumnos de esta investigación, en su plan de estudio no se contempló una asignatura de la antigua diplomatura denominada Lengua instrumental, cuyo descriptor incluía el análisis, producción y valoración del discurso oral y escrito, solución de problemas textuales, corrección reflexiva y valoración textual. Dentro de los problemas textuales y junto a la corrección reflexiva, ocuparía un lugar importante la conciencia ortográfica del futuro docente, sobre todo cuando se ha observado la frecuencia de sus errores ortográficos. Aunque en la literatura existen actividades de escritura muy diversas, para replantear una materia así se proponen algunas prácticas discursivas como las que refleja la Tabla 7. 
Tabla 7. Actividades de escritura.

\section{Prácticas intensivas}

- Cumplimentación de la escala de valoración ortográfica (Rico-Martín, 2003) y reflexión sobre los resultados.

- Detección, explicación y corrección de errores ortográficos en prensa.

- Elaboración de mapas conceptuales previos a borradores de textos sobre una temática elegida y con herramientas virtuales como Coogle, CmapTools o Telarañas. Se pueden proceder a la inversa, elaborar mapas de un escrito dado. En este caso, hay que destacar la utilidad de estos mapas conceptuales para sintetizar y organizar los contenidos de un trabajo escrito, además de poderse realizar de forma colaborativa (Pontes, Serrano \& Muñoz, 2015).

- Separación de párrafos de un texto escrito en un solo párrafo. Empleo de los marcadores textuales y conectores convenientes.

- Análisis y comparación de las características textuales de fragmentos que divergen por el diferente grado de respeto a estas cualidades de coherencia, cohesión, corrección y adecuación.

- Redacción de diversos tipos de citas y referencias bibliográficas según un estilo determinado.

\section{Prácticas extensivas}

- Detección y valoración del uso de la descripción y la exposición en textos de las diferentes áreas del currículo del Grado.

- Análisis de textos con discurso coloquial y transformaciones al académico para proceder después a una revisión colectiva.

- Empleo del lenguaje discursivo hipotético para favorecer las propias representaciones conceptuales de los textos expositivos. Para ello, empleo de textos con verbos en condicional y subjuntivo para introducir nuevas ideas relacionadas con las previas.

- Trabajos de escritura colaborativa realizados en pequeños grupos, en cada uno se llevará a cabo una revisión grupal y, seguidamente se procederá a seleccionar algunos para una revisión colectiva con el grupo clase, se reflexionará sobre la práctica de escritura.

- Elaboración de un monográfico académico según las pautas que aparecen en Rico-Martín (2015).

- Realización de un póster del trabajo monográfico y su ponencia ante el propio grupo de clase como auditorio.

Hay que advertir que conviene que estas propuestas no se limiten a la asignatura mencionada y se integren en tareas de otras materias para que sus resultados sean definitivos.

Esta asignatura se planteó en la antigua titulación vistas las dificultades de redacción de los alumnos, no obstante, si bien en carreras técnicas se han incluido materias cuyo objetivo es trabajar la comunicación oral y escrita, es paradójico que en la formación de maestros se hayan omitido tales contenidos específicos mientras que se incluye la didáctica de estos tipos de comunicación, y nos preguntamos ¿podrán enseñarla aquellos que no la dominan?

Por otra parte, y a favor de la interdisciplinaridad, este estudio concluye, acorde con lo que señalan Marín y Morales (2004), en la necesidad de incluir en las materias de los planes de estudios del Grado la composición frecuente de diferentes textos. Si bien se indican los expositivos y argumentativos como idóneos para el trabajo académico, en opinión de las autoras, un futuro docente debe incorporar a su competencia discursiva una tipología textual más diversa, pues toda ella tiene cabida en el aula escolar; no obstante, el resto de los textos se trabaja más frecuentemente en las asignaturas que dependen del ámbito de conocimiento de la enseñanza de las lenguas. 
Sin embargo, no debe dejarse como responsabilidad única de los profesores de Lengua, es fundamental desechar la creencia de que leer y escribir concierne solo a estos especialistas y adoptar la premisa de que en la competencia escrita de los alumnos deben intervenir coordinados todos sus docentes, cualquiera que sea su ámbito científico (Carlino, 2003), tal como subyace en el movimiento anglosajón Writing across the curriculum, de forma que se supere la excesiva compartimentalización de los conocimientos propia del ámbito universitario (Nigro, 2006; Porto, 2008). 


\section{REFERENCIAS BIBLIOGRÁFICAS}

Adam, J. M. (1999). Linguistique textuelle. Des genres de discours aux textes. París: Nathan/ HER.

Argáez, S. F., Fernández, M. E., Martínez, M. I., Sandoval, L. K. \& Ortiz, M. (2010). Hábitos de redacción en estudiantes universitarios. Revista UABC, 69, 24-37.

Arnoux, E., Di Stefano, M. \& Pereira, C. (2002). La lectura y la escritura en la universidad. Buenos Aires: EUDEBA.

Bajtín, M. (1979). Estética de la creación verbal. México: Siglo XXI.

Barboza, F. D., Aguirre, R. \& Peña, J. (2008). Una experiencia para estimular la composición escrita en estudiantes universitarios de formación docente. Educere, 40, 101-108.

Carlino, P. (2003). Leer textos científicos y académicos en la educación superior: Obstáculos y bienvenidas a una cultura nueva. Uni-pluri/versidad, 3(2). Universidad de Antioquia (Medellín, Colombia) [en línea]. Disponible en: http://aprendeenlinea.udea.edu.co/revistas/index.php/unip/article/ viewFile/12289/11146

Carlino, P. (2007). ¿Qué nos dicen hoy las investigaciones internacionales sobre la escritura en la universidad? Ponencia presentada en el I Encuentro Nacional sobre políticas institucionales para el desarrollo de la lectura y la escritura en la educación superior. ASCUN y Red de discusión sobre lectura y escritura en la educación superior. Universidad Sergio Arboleda, Bogotá (Colombia) [en línea]. Disponible en: http://www.ascun-redlees.org/2007/01/21/primerencuentro-nacional-sobre-politicas-institucionales-para-el-desarrollo-de-lalectura-y-escritura-en-la-educacion-superior/

Cassany, D., Luna, M. \& Sanz, G. (2000). Enseñar lengua. Barcelona: Editorial Graó.

Castelló, M., Pardo, M. \& Fuentealba, M. O. (2011). Ensenyar a escriure textos acadèmics a la Universitat: El guiatge i la revisió col.laborativa del projecte de recerca en els estudis de Psicologia. Aloma, 29, 131-152.

Catuogno, M., Mallo, A. \& Rivarola, M. (2003). La expresión escrita de los jóvenes universitarios. Kairos, 12, 1-13 [en línea]. Disponible en http://www.fices. unsl.edu.ar/ kairos/k12-02.htm

Codorniú-Raga, M. J. \& Vigil-Colet, A. (2003). Sex differences in psychometric and chronometric measures of intelligence among young adolescents. Personality and Individual Differences, 35, 681-689. 
Colom, R. \& García-López, O. (2002). Sex differences in fluid intelligence among high school graduates. Personality and Individual Differences, 32, 445-451.

Dolz, J., Gagnon, R., Mosquera, S. \& Sánchez Abdchi, V. (2013). Producción escrita y dificultades de aprendizaje. Barcelona: Graó.

Echavarri, M., Godoy, J. C. \& Olaz, F. (2007). Diferencias de género en habilidades cognitivas y rendimiento académico en estudiantes universitarios. Universitas Psychologica, 6(2), 319-329.

Marín, E. I. \& Morales, O. A. (2004). Análisis de textos expositivos producidos por estudiantes universitarios desde la perspectiva lingüística discursiva. Educere, $26,333-345$.

Martí, J., Amat, A. \& Jiménez, I. (2013). El uso de evidencias en la escritura científica en los estudiantes de magisterio. Textos de Didáctica de la Lengua y de la Literatura, 64, 40-46.

Medina, A. M. (1994). La enseñanza de la ortografía en la universidad. REALE, 2, 73-78.

Mesa, M. C. (2000). Motivación hacia el aprendizaje y estilos atributivos sobre las causas del éxitol fracaso escolar en un contexto multicultural. Tesis doctoral inédita, Universidad de Granada, Granada, España.

Mesa, M. C. \& Sánchez, S. (1996). Educación y situaciones bilingües en contextos multiculturales. Estudio de un caso: Melilla. Granada: Laboratorio de Estudios Interculturales y CIDE.

Milian, M. (2001). Interacción de contextos en la investigación sobre composición escrita. En A. Camps (Coord.), El aula como espacio de investigación y reflexión. Investigaciones en didáctica de la lengua (pp. 23-36). Barcelona: Graó.

Morales, O. A. \& Espinoza, N. (2005). El desarrollo de la escritura de estudiantes universitarios. Lectura y vida: Revista latinoamericana de lectura, 26(1), 26-37.

Morales, O. A. \& Hernández, L. G. (2004). Estudio descriptivo del uso de la ortografía de los estudiantes universitarios de nuevo ingreso. Kaleidoscopio, 1(2), 151-159.

Nigro, P. (2006). Leer y escribir en la universidad: Propuestas de articulación con la escuela media. Educación y Educadores, 9(2), 119-127.

Oliveras, B. \& Sanmartí, N. (2011). Diferentes usos para la misma agua. Autorregulación del proceso lector. Producción de un texto narrativo. Leer.es, 9, 1-13 [en línea]. Disponible en: http://leer.es/wp-content/uploads/publicaciones/ PDFs/201109.pdf 
Pontes, A., Serrano, R. \& Muñoz, M. (2015). Los mapas conceptuales como recurso de interés para la formación inicial del profesorado de enseñanza secundaria: Opiniones el alumnado de ciencias sociales y humanidades. Educación XX1, 18(1), 99-124.

Porto, M. (2008). Evaluación de estudiantes universitarios: ¿Toma de decisiones colegiada? Cuadernos FHyCS-UNJu, 34, 133-149.

Rico-Martín, A. M. (2003). ¿Qué opinan los futuros docentes acerca de su ortografía? Revista Electrónica Interuniversitaria de Formación del Profesorado 6(1), 1-9 [en línea]. Disponible en: www.aufop.org/publica/reifp/articulo. asp?pid $=213 \&$ docid $=1054$

Rico-Martín, A. M. (2015). Cómo redactar un trabajo académico [en línea]. Disponible en: http://es.slideshare.net/AnaMRicoMartn/cmo-redactar-un-trabajoacadmico-ana-m-ricomartn

Robledo, W. \& Ayllón, S. (2003). Estudios sobre la predictibilidad de los 5 subtests del DAT (Informe de investigación). Córdoba (Argentina): Universidad Empresarial Siglo 21.

Rodríguez, C. \& Ruiz Bikandi, U. (2013). El discurso científico en el aula. Textos de Didáctica de la Lengua y de la Literatura, 64, 5-8.

Rodríguez, D. \& Valldeoriola, J. (2009). Metodología de la investigación. Barcelona: UOC.

Sánchez, S. (Dir) (2010). El abandono escolar temprano en las ciudades de Ceuta y Melilla. Madrid: Ministerio de Educación.

Sánchez Abchi, V., Diuk, B. \& Borzone, A. M. (2009). El desarrollo de la escritura de palabras en español: Interacción entre el conocimiento fonológico y ortográfico. Interdisciplinaria, 26(1), 95-119.

Sánchez Avendaño, C. (2005). Los conectores discursivos: Su empleo en redacciones de estudiantes universitarios costarricenses. Filología y Lingüistica, 31(2), 169199.

Sánchez Avendaño, C. (2006). ¿Cuestión de método? Sobre los cursos remediales universitarios de expresión escrita. Revista de Educación, 30(1), 65-81.

Sánchez Lozano, C. (2007). La escritura ortográfica en estudiantes universitarios. Textos de didáctica de la lengua y la literatura, 45, 99-106.

Tamayo, O. E. \& Sanmartí, N. (2005). Características del discurso escrito de los estudiantes en clases de ciencias. Revista Latinoamericana de Ciencias Sociales, Niñezy Juventud, 3(2) [en línea]. Disponible en http://revistaumanizales.cinde. org.co/index.php/Revista-Latinoamericana/article/viewArticle/301 
Tapia, M., Burdiles, G. \& Arancibia, B. (2003). Aplicación de una pauta diseñada para evaluar informes académicos universitarios. Revista Signos. Estudios de Lingüistica, 36(54), 249-257 [en línea]. Disponible en: http://www.scielo.cl/ scielo.php?script $=$ sci_arttext\&pid=S0718-09342003005400009\&lng $=$ es\&tl ng=es. 10.4067/S0718-09342003005400009

Weissa, E. M., Kemmlera, G., Deisenhammerb, E. A., Fleischhacker, W. \& Delazerc, M. (2003). Sex differences in cognitive functions. Personality and Individual Differences, 35, 863-875.

\section{NOTAS}

1 Este trabajo forma parte del proyecto de investigación "Estudio de la competencia multilingüe del alumnado universitario en el Campus Universitario de Melilla, incluido en el II Contratoprograma de investigación de la Facultad de Educación y Humanidades de Melilla. Plan 20 (20122014)", financiado por el Vicerrectorado de Política Científica e Investigación de la Universidad de Granada (España).

2 Fuente: Instituto Nacional de Estadística (INE). Disponible en http://www.ine.es

3 Aunque somos conscientes de que el empleo de la denominación 'de origen peninsular o europeo' puede dar lugar a dudas, en estudios españoles es frecuente este uso para referirse al sector poblacional de las ciudades españolas ubicadas fuera de la Península Ibérica que, en un primer momento, procedió de ella. Fuera de las islas españolas, se utiliza, sobre todo, para distinguirlo del grupo de origen bereber, de procedencia africana.

Para conocer mejor las características sociolingüísticas de la población melillense, véase RicoMartín, A. M. (2006). Breve perspectiva de la enseñanza del español como lengua extranjera o segunda lengua en Melilla. En Instituto Cervantes, Enciclopedia del español en el mundo. Anuario del Instituto Cervantes 2006-2007 (pp. 440-441). Madrid: Instituto Cervantes. 